\title{
Trajectory Assessment and Modification Tools for Next Generation Air Traffic Management Operations
}

\author{
Connie Brasil, Paul Lee, Matthew Mainini, Jeffery Homola \& Hwasoo Lee, San Jose State University, \\ Moffett Field, CA \\ Thomas Prevot \& Nancy Smith, NASA Ames Research Center, Moffett Field, CA
}

\begin{abstract}
This paper reviews three Next Generation Air Transportation System (NextGen) based high fidelity air traffic control human-in-the-loop (HITL) simulations, with a focus on the expected requirement of enhanced automated trajectory assessment and modification tools to support future air traffic flow management (ATFM) planning positions. The simulations were conducted at the National Aeronautics and Space Administration (NASA) Ames Research Center's Airspace Operations Laboratory (AOL) in 2009 and 2010. The test airspace for all three simulations assumed the mid-term NextGen En-Route high altitude environment utilizing high altitude sectors from the Kansas City and Memphis Air Route Traffic Control Centers.

Trajectory assessment, modification and coordination decision support tools were developed at the AOL in order to perform future ATFM tasks. Overall tool usage results and user acceptability ratings were collected across three areas of NextGen operations to evaluate the tools. In addition to the usefulness and usability feedback, feasibility issues, benefits, and future requirements were also addressed. Overall, the tool sets were rated very useful and usable, and many elements of the tools received high scores and were used frequently and successfully. Tool utilization results in all three HITLs showed both user and system benefits including better airspace throughput, reduced controller workload, and highly effective communication protocols in both full Data Comm and mixed-equipage environments.
\end{abstract}

\section{Introduction}

According to the Federal Aviation Administration's (FAA) airspace capacity forecast, demand for air travel is returning, and passenger traffic is expected to double in the coming decades [1]. In the United States and Europe, a fundamental shift is taking place in air traffic control systems and procedures to accommodate and better manage this growth. The vision of the Next Generation Air Transportation System (NextGen) in the United States and Single European Sky ATM Research (SESAR) in Europe depends on managed as opposed to controlled airspace, with aircraft operators, air navigation service providers and airports working collaboratively to execute optimized air traffic operations [2-4]. Aided by enhanced automation and decision support tools (DSTs), the practice of air traffic flow management (ATFM) will support increased airspace capacity with more efficient air traffic control operations. Aircraft equipped with automatic dependent surveillance-broadcasting (ADS-B), performance-based navigation and controller/pilot data link communications (Data Comm) are expected to contribute to and to take advantage of a more efficient system. In order to service these better equipped aircraft appropriately, the ATFM ground systems will need to be enhanced and adaptable as well.

The Airspace Operations Laboratory (AOL) has developed and evaluated a high-fidelity, adaptable ground based simulation software platform called Multi Aircraft Control System (MACS) [5, 6]. Prototyped into this test bed is a suite of trajectory assessment and modification tools used for future flow-based trajectory management (FBTM), a process for planning and coordinating aircraft trajectory changes to meet local area traffic flow management objectives. This tool set includes situation awareness tools as well as planning and coordination tools. In conjunction with the FAA, the NASA AOL has simulated, defined and enhanced the FBTM tools in different operational environments envisioned for NextGen. The purpose of this paper is 
to review the various situational and planning tools used with aircraft trajectory assessment and modifications, demonstrate the usefulness and usability of such tools and show the feasibility and benefits of a defined set of FBTM tools as they relate to future air traffic control enhanced ground based implementation.

\section{Background}

The first of the three studies was the 2009 MultiSector Planning (MSP2) HITL that investigated adding a new ATFM position to the Air Route Traffic Control Centers (ARTCC) facility team [7-9]. The members of this team included Traffic Management Coordinators (TMCs), Area Supervisors, and Radar Controllers as well as a newly defined Multi-Sector Planning position which was staffed by planning team members that had both Area Supervisor and TMC experience. The MSP position was designed to fill a gap between the TMC's strategic/regional operations and the more locally focused operations of the Area Supervisors. This initial study introduced a suite of planning tools for flow-based trajectory management operations that included tools for traffic load and complexity assessment as well as trajectory planning and coordination utilizing a fully Data Comm operational environment.

The second study was a follow-on MSP HITL (MSP3) completed in 2010 [10, 11]. This study incorporated the FBTM tool set into the Area Supervisor and TMC workstations and looked at how these advanced planning tools could provide assistance in a more near-term mixed-equipage environment where not all aircraft are Data Comm equipped. The FBTM tools, which had originally been designed for integrated Data Comm equipage, were improved to accommodate unequipped aircraft that had to be controlled via voice communication. Planning workstations were enhanced with equipagesensitive color-coding of data tags and aircraft symbols, as well as waypoint-based trajectory planning and amendment mechanisms that were suitable for voice clearances. The workstations used by TMCs and Area Supervisors were also enhanced with equipage sensitive aircraft filters and traffic complexity computations as well as trajectory planning and communication mechanisms compatible with those used by the sector controllers.
In order to demonstrate a broader NextGen application of the planning tool set, a separate third HITL, also completed in 2010, was conducted demonstrating the benefits and feasibility of Flexible Airspace Management (FAM) [12-16]. The FAM concept allows for dynamic airspace reconfiguration to balance traffic and weather demands across multiple sectors and Areas of Specializations. In addition to the newly designed reconfiguration tools, FAM relies heavily on strong trajectory assessment and modification tools with not only advanced air-toground Data Comm but ground-to-ground procedures as well.

The FAA has laid the groundwork for future automation and increased DST technologies with their NextGen implementation plan. The plan calls for seven different solution sets to meet and exceed their implementation goals [2]. The NASA AOL HITLs address two of them; Trajectory Based Operations (TBO) and Collaborative Air Traffic Management (CATM) [2]. The TBO solution set focuses primarily on the high altitude cruise portion of en-route operations, incorporating capabilities, DSTs, and automation to manage aircraft flight paths by trajectory to ensure full performance based navigation and accommodate user preference. The CATM solution focuses on improving the systemwide traffic flow management capabilities. Advanced automation systems will work together to provide consistent airspace capacity solutions, user preferred routing options, and dynamic airspace sector boundaries for more flexible air traffic control management. Both the MSPs and FAM HITLs address the decision support tools and automation required to fulfill the NextGen plan. The FBTM tools were designed to provide a more flexible airspace management environment for the ground operations as well as accommodate flight operator user preferences.

\section{Trajectory Assessment and Modification Tools}

The trajectory assessment and modification FBTM tools were built into the planning stations of all participants in the ATFM team, including the TMCs, Area Supervisors, and Radar Controllers. This paper will focus on the planning tools for the TMC and Area Supervisor positions. Across all three HITLs there were 14 operationally current, ATFM 
participants. Five participated as Area Supervisors, five were TMCs, and four as MSPs; MSP participants had operational experience as both Area Supervisors and TMCs. FBTM tools for situation assessment, planning and plan coordination were distributed amongst the planning stations of each team member to enable a common understanding of the current situation in order to assess available options for the management, communication and execution of proposed plans [7]. Figure 1 show the typical planning station used in all three HITLs and illustrates many of the FBTM capabilities. Most of these tools (orange text) were developed and tested in the 2009 MSP HITL. Tools highlighted in yellow were added or enhanced during the 2010 MSP HITL and the boundary edit window highlighted in green was added for the FAM 2010 HITL.

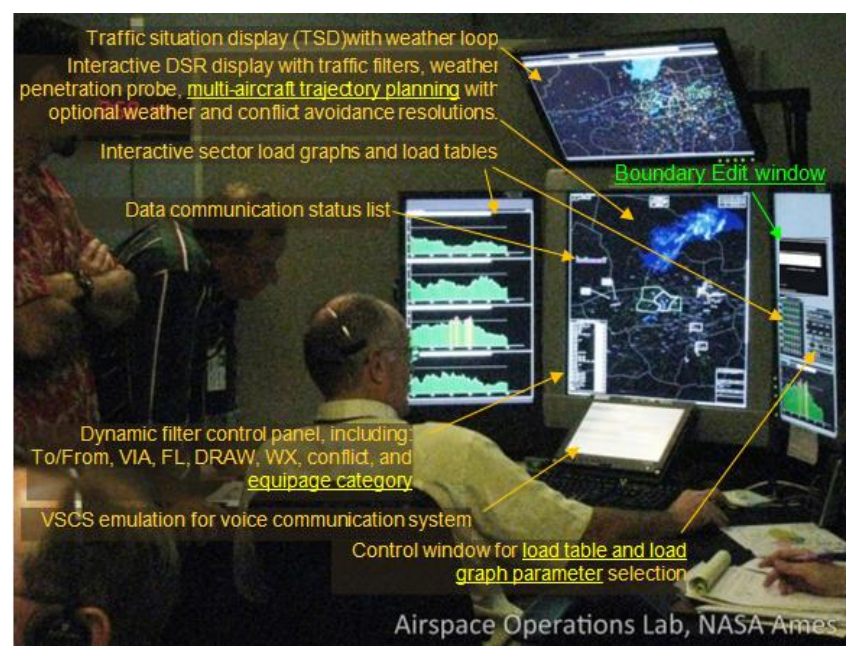

\section{Figure 1. AOL ATFM Planning Station}

The ATFM workstation includes tools designed to assess and manage traffic complexity in a trajectory based operations (TBO) environment. Trajectory predictions drive the situation assessment tools and planning team members enact changes by modifying and communicating trajectories for aircraft to other planning stations for review and to the tactical controllers for execution. The assessment tools include the load display control window, load table window, load graph window, and the aircraft (AC) filters. Load assessment tools are located to either side of the planner positions main Display System Replacement (DSR) display and the AC filter is located within the DSR display itself, as shown in
Figure 1. All of these situation assessment planning tools interact with each other.

Traffic assessment tools are also interactive with the trajectory modification tools. The modification tools are used in creating and analyzing actual solutions or trial plans. These trial plans can be looked at for single or multi-aircraft trajectory planning as well as Data Comm-based coordination of trajectories between ATFM operators. All trajectory modifications are done via the main DSR Display, which has a highly interactive trial planning tool that allows the user to look at all aircraft in their airspace and the surrounding airspace. The display allows the user to filter the traffic to highlight various subsets of aircraft, for example, they can look at traffic predicted to penetrate weather cells. Users can look at any aircraft's route of flight and trial plan a solution (or multiple solutions) for any particular problem. In addition to the interactive filtering and trial planning options, the DSR has full air-ground and ground-ground Data Comm capability. Both route and altitude trial plans can be data linked directly to the aircraft flight deck, the radar controllers responsible for that aircraft, or to other planning team members to preview a potential trial plan solution.

\section{Situation Assessment Tools}

In order to support the operators' assessment of traffic volumes, congestion points, and weather impacts within a large congested airspace, new traffic load, sector complexity and aircraft filtering tools were prototyped and tested. Each of the three HITL's scenarios included very high traffic congestion and/or severe convective weather. ATFM team members were asked to keep sector loads (aircraft count) at or below a specified monitor alert parameter (MAP). In today's ATFM environment, the MAP is used to trigger notification, via the enhanced traffic management system (ETMS), that sectors efficiencies may be impacted during a specific time parameter. Typical MAP values for current air traffic control operations range from 5 to a maximum of 18 aircraft in a single sector during a specific period of time [17]. Due to the enhanced automation DSTs, along with the full Data Comm and mixed-equipage environment, the MAP values in the three HITLs were increased and ranged from 22 to 26 . 


\begin{tabular}{|l|l|l|}
\hline \multicolumn{2}{|l|}{ Table 1. AC Filter Options } \\
\hline Filter Option & CRD/Keyboard Filter Command FC & Filter Definition \\
\hline TO & FC TO [airport] or [ARTCC] & Filter aircraft to specific arrival airport(s) \\
\hline FROM & FC FROM [airport] or [ARTCC] & Filter aircraft coming from a specific airport(s) \\
\hline VIA & FC VIA [fix] & Filter aircraft going via a certain waypoint/fix \\
\hline FL (ALT.) & FC FL [alt] [alt] & Filter aircraft by altitude(s) \\
\hline GEO (SECTOR) & FC GEO [ZKC90] or [ZME] [T] & Filter aircraft by sector ownership or ARTCC @ Time X \\
\hline DRAW or LINE & FC DRAW or LINE [F1] [T15=35] & Filter aircraft that will enter any “Draw Tool” defined area @ Time X \\
\hline WX 1, 2,3 & FC WX 1,2,3 [T25-45] & Filter aircraft that are predicted to go into weather low (1), medium (2), and high (3) @ Time X \\
\hline CONFLICT & FC CON T1=30 & Filter aircraft that are predicted to be in conflict at Time X (T1-30, between now and 30 minutes) \\
\hline ACID & FC ID [NWA123] & Filter aircraft by ID (NWA123) \\
\hline AIRLINE & FC AIRLINE [SWA] & Filter aircraft by airline (SWA) \\
\hline AIRPORT & FC AIRPORT [DFW] & Filter aircraft to/from this airport \\
\hline DIR & FC DIR [Heading Range 045-090] & Filters aircraft heading in a specific direction \\
\hline LOAD & FC LOAD & Filter aircraft based on Load Table/Graph criteria selected \\
\hline FR & FC FR IFR or FC FR TFR & Filter aircraft based on equipage: Fully Equipped and Unequipped \\
\hline
\end{tabular}

In order to gain awareness of the current and predicted traffic situations, new dynamic aircraft filter capabilities were prototyped allowing operators to highlight only those aircraft that share a particular property; for example, traffic can be filtered such that only aircraft that fly to or from specific airports, or via designated routes, waypoints, or altitudes are displayed. The tool can also highlight aircraft that pass through specific sectors, dynamically drawn objects or forecasted convective weather areas. Filters can be combined, dynamically added, deleted or edited and color coded. Aircraft that do not pass the filter test are dimmed into the background, aircraft that meet the selected critera are highlighted. Table 1 lists all the AC filter options available to sort and assess traffic flows. The AC filter window also works in combination with the traffic load and complexity tables/graphs to display only the aircraft that correspond to the predicted load and complexity table/graph values.

Similar to ETMS today, traffic loads for sectors are computed as the number of aircraft predicted to be in the sector for a given time frame. The results are presented in tables and graphs. Operators can use the load tables or graphs to select a value for a specific time and sector, and the aircraft contributing to that value are are highlighted on the display. In order to account for complexity factors that go beyond a simple aircraft count, the tables/graphs can be changed to show other parameters, such as number of unequipped or transitioning aircraft, aircraft predicted to be in conflict, or aircraft predicted to penetrate weather hazards. In addition to these values, a realtime estimate of the sector complexity is also computed. The complexity calculation includes the factors described above as well as the sector shape and size. Therefore, operators can use the complexity values instead of the total number of aircraft (MAP value) to provide a more accurate estimate of the workload within any given sector. Figure 2 shows a close up view of the load table with complexity values.

All load table/graph values reflect active trajectories. Predictions for provisionial trajectories are also shown when new trajectory trial plans are visible. All values are color coded with reference to the MAP/complexity threshold: green indicates that traffic is below the threshold, yellow is at the threshold, and red indicates that the threshold has been exceeded. Cyan blue numbers represent predicted values based on any open trial plan trajectories. 


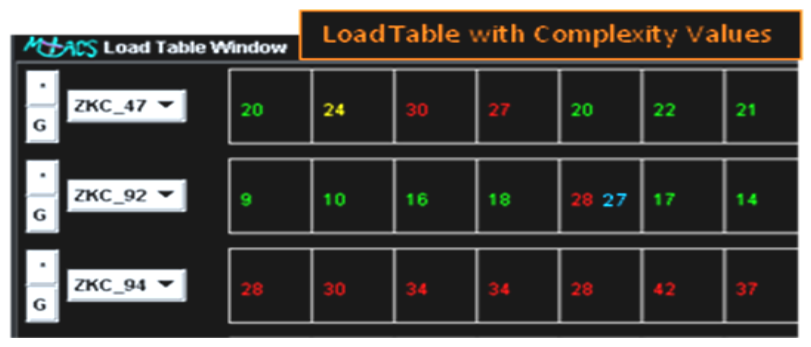

Figure 2. Load Table with Complexity Values

Figure 3 shows the planning station layout of all the load and complexity tables/graphs. ${ }^{1}$

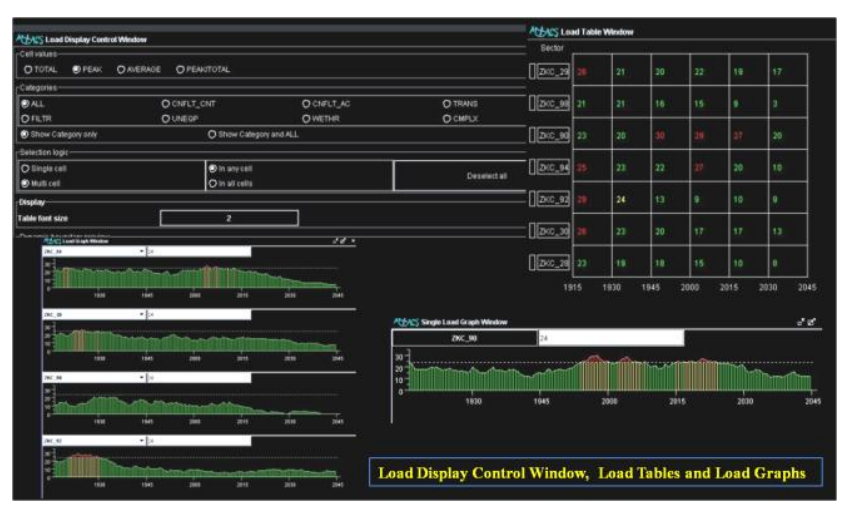

\section{Figure 3. Load and Complexity Assessment Tools}

In addition to the AC filters and traffic load and complexity tables/graphs, new tools were developed in the FAM HITL to incorporate the flexible airspace redesign concept into the suite of FBTM situational assessment tools [12-16]. The airspace design tools allow the planners to modify existing sector boundaries either manually or by predefined algorithm generated configurations. As airspace changes are being made, real-time feedback is provided to the planner via the tables/graphs, as to how the modifications impact sector load and complexity values. Figure 4 shows an algorithmgenerated airspace configuration and the traffic load tables/graphs updated to reflect its impact on the traffic load for each test sector.

Once the traffic situation is assessed, the planner then needs to take action to solve any traffic overload, airspace congestion, or weather avoidance issues. Utilizing the highly interactive trajectory modification and coordination tools, the planner

${ }^{1}$ Refrences 7-14 give a complete and detailed description of all FBTM tools used in all three HITLs could quickly develop flight plan solutions that complement the planned airspace configuration, then distribute the solution to other members of the ATFM team. With these paired operations, closely coordinated demand (traffic flow) and capacity (airspace) adjustments can be performed, illustrating how the FBTM tools can support other operations.

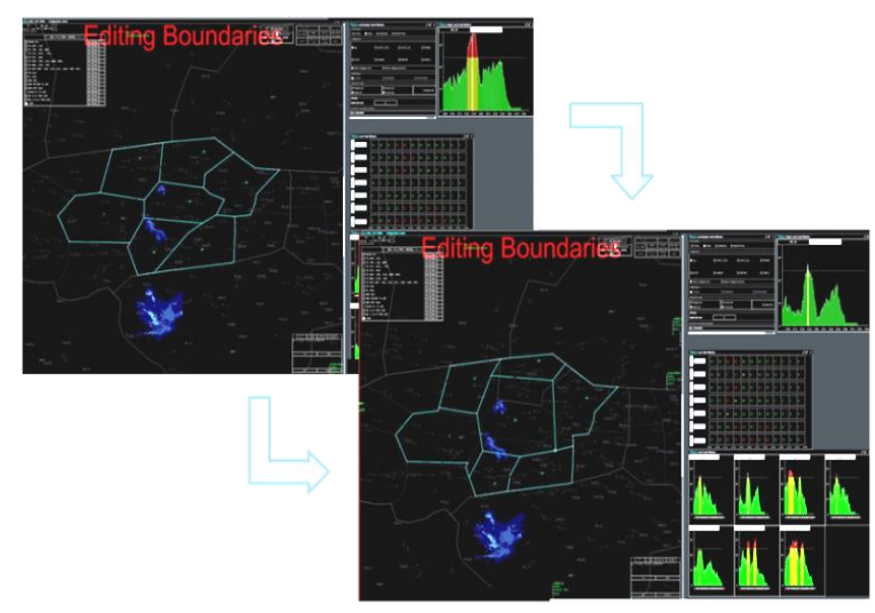

Figure 4. Flexible Airspace Design Tools

\section{Trajectory Planning and Coordination Tools}

The tool set for creating and analyzing the actual solutions, or trial plans, include some of the same tools discussed in the situational assessment sections above. The tools in this section will be discussed in terms of their use in the trajectory planning of a problem set of aircraft. The Traffic Situation Display (TSD), DSR traffic view display, load and complexity tables/graphs, AC filters, and the groundto-ground Data Comm system were all used to help the planning team solve load problems or design reroutes for weather situations and then distribute those solutions to the appropriate team members.

The TSD was located above the operator and was used to show the relative direction of all the traffic, any trial plan routes that were developed, and to display the current and future forecasted weather information. The TSD in this simulation did not have all the full capabilities it has in the field and was instead used to provide a more general overview of the traffic flows and the weather, and therefore, will not be discussed in detail. 
The DSR traffic situation display was the main interactive device used by the ATFM team to look at and solve various traffic re-routing situations. The DSR is host to a highly interactive trial planning tool that allows the user to look at all aircraft in their airspace and the surrounding airspace. They can filter the aircraft to look at various subsets of traffic, traffic predicted to go into weather cells, and any aircraft's route of flight to trial plan a solution or multiple solutions to help with any particular problem. In addition to all the interactive filtering and trial planning options, the DSR has full airground and ground-ground Data Comm capability. Both route and altitude trial plans can be data linked directly to the aircraft flight deck, to the controllers responsible for that aircraft, or to other planning team members to preview a potential trial plan solution. Figure 5 shows an example of the DSR traffic view display with weather information, multiple aircraft selections, AC filter, and the Data Comm status list.

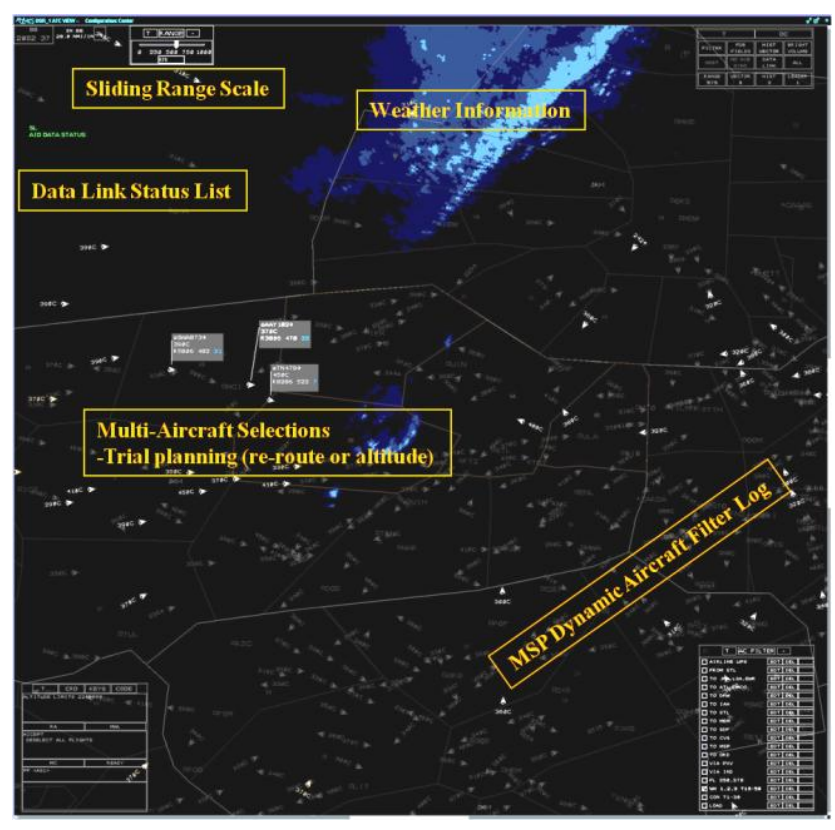

\section{Figure 5. Example of DSR Traffic View Display}

The interactive load and complexity tables/graphs are used by the planners to monitor and locate where traffic load and complexity situations (i.e., weather, airspace/airport congestion points or transitioning aircraft) may be occurring in their areas. The load tables/graphs are completely interactive with the DSR display for trial planning, allowing the user to identify preferred re-routing candidate aircraft in their airspace. They can use the load tables/graphs to look at sectors with predicted high peak traffic loads or high complexity values or filter the load graphs to look at various subsets of predicted traffic loads. In addition, the interactive load graphs allow the user to identify aircraft that are predicted to contribute to traffic load peaks at specific time intervals, down to the minute. Figure 6 shows an example of the load graph with the first peak period highlighted to show only those aircraft that are affecting that peak time interval.

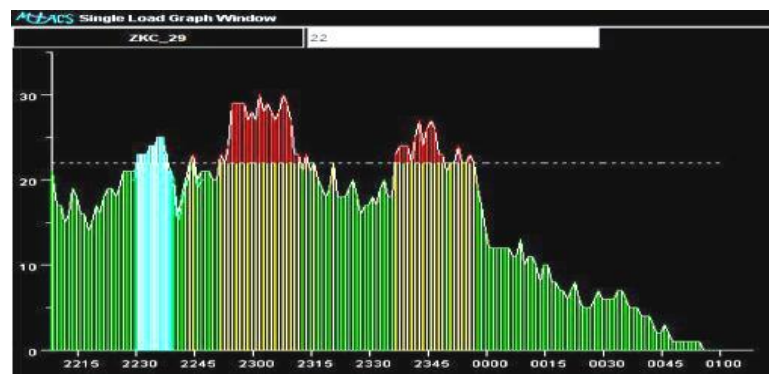

Figure 6. Load Graph with First Peak Highlighted

The AC filter function is used to control the display of the load and complexity table/graph data onto the DSR. As described above, the AC Filter Window is located on the DSR display and enables the user to select and filter the aircraft that are highlighted and displayed on the DSR in order to define problem areas or perform trial plan solution ideas on specific subsets of traffic. The filters work by categories which are defined by keywords (see Table 1). As can be seen in Figure 7, these keywords are the first word of each row in the AC filter window. The user can chose from the predefined and/or custom filters, or make their own and color code them as needed.

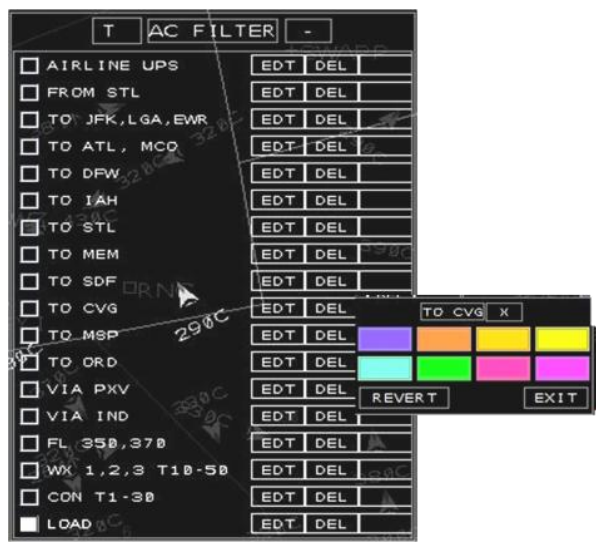

Figure 7. AC Filter Window with Color Coding 
Once the candidate aircraft are identified, the DSR planning tools are used to develop solutions and build trial plans. There are a variety of ways to enter the trial plan mode: the user can chose one aircraft to trial plan or make a multiple aircraft selection to build a trial plan for a group of aircraft, the user can type commands into the DSR command readout display, or use the interactive flight data block (FDB) trial plan features. The typed commands include FF (selects aircraft for group trial planning), TT (opens basic route trial plan), TA (opens an altitude trial plan), and TR (opens a more specified route trial plan). The data block requires picking on specific fields within the FDB, which include: the arrow next to the aircraft call sign (opens basic route trial plan, similar to TT command); the same arrow (but magenta-colored) to review a suggested trial plan from another planner or a trial plan already sent to others; the altitude line of the FDB (opens an altitude trial plan, similar to the TA command); the conflict number to start a automated trial plan resolution to solve for the predicted traffic conflict; and the weather number to start a trial plan to solve for the predicted weather penetration. Table 2 gives a full definition of all the trial plan options available with both keyboard and interactive FDB commands.

Table 2. Trial Plan Options

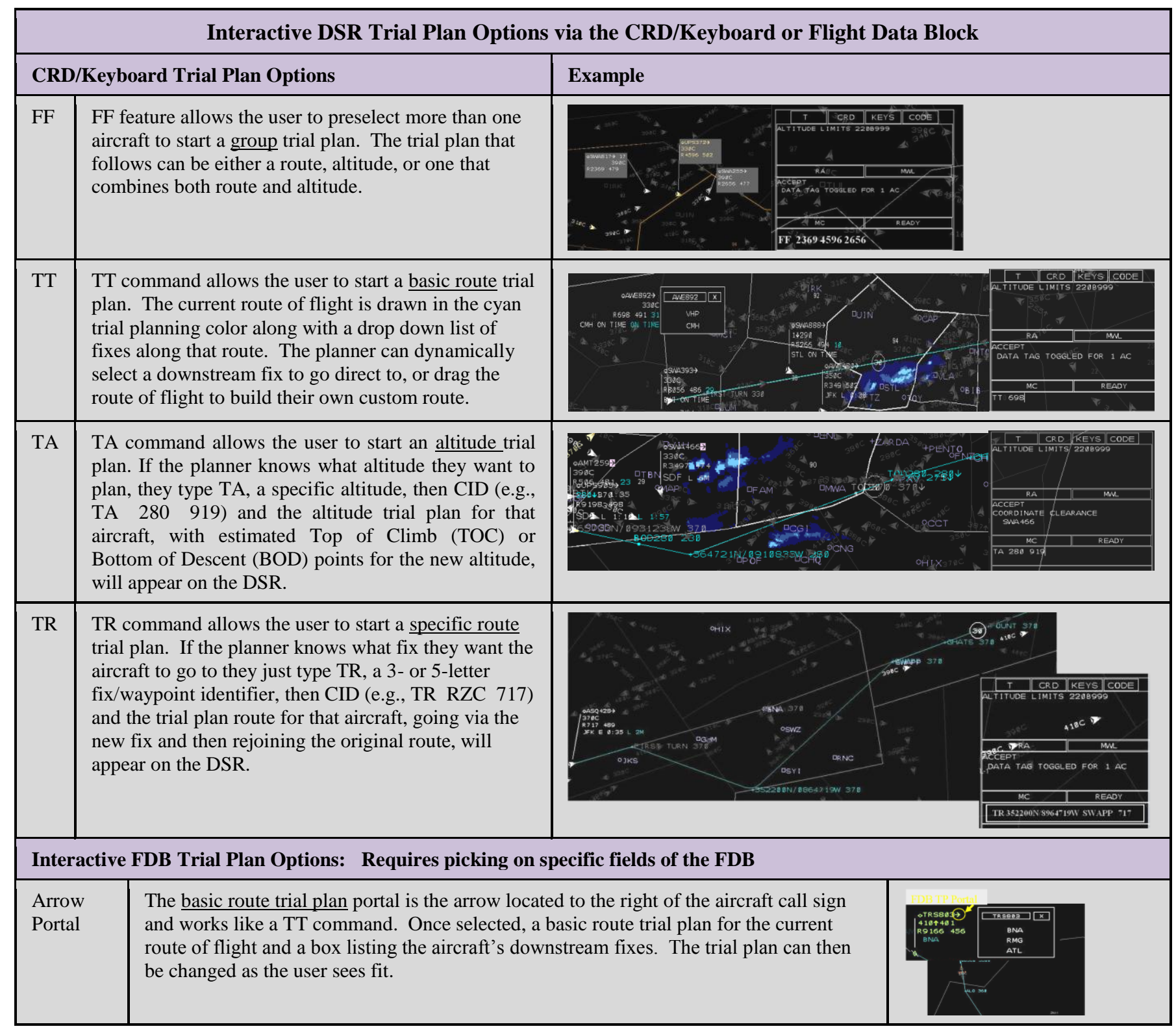




\begin{tabular}{|c|c|c|}
\hline $\begin{array}{l}\text { Arrow } \\
\text { Portal } \\
\text { Magenta }\end{array}$ & $\begin{array}{l}\text { The arrow turns a magenta color when there is a suggested trial plan from another } \\
\text { planner or after the user has sent a trial plan via Data Comm to another user. To review } \\
\text { a suggested trial plan received from another planner, or to see a trial plan already sent, } \\
\text { click on the magenta arrow and the route will appear in magenta color. }\end{array}$ & 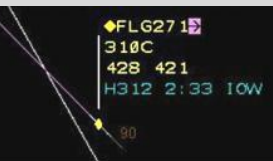 \\
\hline $\begin{array}{l}\text { Altitude } \\
\text { Line }\end{array}$ & $\begin{array}{l}\text { The altitude portion of the FDB starts an altitude trial plan. This differs from a TA in } \\
\text { that the user then selects an altitude to trial plan from a drop down list }\end{array}$ & 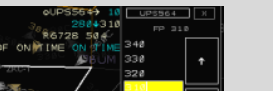 \\
\hline $\begin{array}{l}\text { Conflict } \\
\text { Time-to- } \\
\text { go } \\
\text { Number }\end{array}$ & $\begin{array}{l}\text { The conflict number is located to the right of the trail plan portal arrow and shows how } \\
\text { many minutes until the aircraft will lose separation with another aircraft. Picking on the } \\
\text { number will open a trial plan to allow the user to find a solution for the conflict problem }\end{array}$ & \begin{tabular}{|l|}
$\triangle A C A 625 \rightarrow 5$ \\
$380 C$ \\
$246 \quad 394$
\end{tabular} \mid \\
\hline $\begin{array}{l}\text { Weather } \\
\text { Time-to- } \\
\text { go } \\
\text { Number }\end{array}$ & $\begin{array}{l}\text { The weather number shows how many minutes until the aircraft are predicted to } \\
\text { penetrate weather and is located in the third line of the FDB next to the indicated } \\
\text { airspeed. Picking on the number will open a trial plan to allow the user to find a solution } \\
\text { for the weather penetration problem. }\end{array}$ & 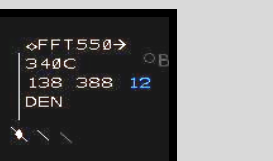 \\
\hline
\end{tabular}

When a resolution is found, the trial plan solution can be shared with any of the ATFM planning stations, including the radar controllers, for review and implementation. Using Data Comm, a simple command can send an entire selection of trajectories to different planning stations. The receiving planners can then review the plan on their own DSR using the situation assessment tools and modify, approve or decline the suggested trajectory change. Utilizing the uplink clearance (UC) command, the users can uplink the trajectory modification directly to the aircraft, coordinate the clearance (CC command) with the radar controllers who have track control of those aircraft, or send a modified coordination plan (CP command) to other team members. Similarly, the dynamic boundary changes that occurred in the FAM HITL, could also be shared from one station to another and then when approved by the planning team, sent to the radar controller for implementation. In addition to the NextGen Data Comm protocols incorporated in these three HITLs, air-ground voice communication capability was also maintained with all aircraft during the simulations. MSP2 and FAM were both full Data Comm equipped environments with voice available as needed, whereas, MSP3 was a mixed communication equipage simulation encompassing both Data Comm and non-Data Comm equipped aircraft.

The collaborative use of the situational awareness, trajectory modification and interactive FBTM tools for plan coordination can go a long way towards the goal of the NextGen midterm operations. The following section gives an overview of the usage results of all the FBTM tools and the ratings given for usefulness and usability across all three HITLs.

\section{Results}

This results section presents a high level combined usage summary of all the FBTM tools across all three HITLs, followed by a combined overview of all the usefulness and usability ratings from all the ATFM participants in each of the three studies. Usage data and post-simulation questionnaire data were taken for all of the various tools and details can be found in References 9-13. A summary of the major results will be given here. Following each results section, will be a review of the relevant experimenter observations, ratings results, and additional participant input.

\section{Trajectory Assessment and Modification Tool Usage Data}

In general the load and complexity tables/graphs, AC filters, trajectory planning and coordination tools were used quite often. As the scenarios in each of the HITLs were designed to exceed high peak and complexity levels, the planners were, by design, obligated to use all the automation tools at their disposal to bring the traffic loads under control with limited disruption of the user preferred 
routing in which all aircraft were flying. The goal was to increase aircraft throughput without increasing ATFM or controller workloads.

Load and complexity tables/graphs were used throughout each HITL as indicated in Figure 8. Overall, the ATFM team used the Load Tables $(64.5 \%)$ more than the Load Graphs $(35.5 \%)$. The load table was considered more necessary for the traffic assessment and planning tasks than the load graphs, but the graphs were still valuable. The questionnaire results show that the graphs were used as a reference for how long the peak period would last and to immediately see which aircraft were contributing to the traffic load. One Area Supervisor said that the graphs were "excellent at getting exact aircraft that will affect the sector at a given minute." Another commented the Load Tables were "essential in deciding how to help/plan sector load mitigation."

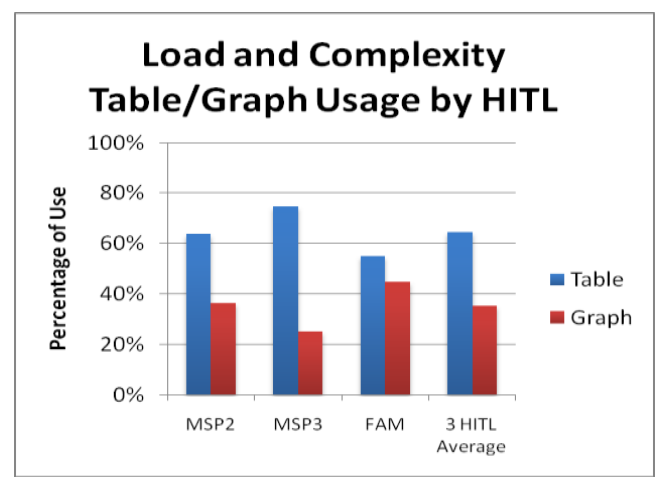

\section{Figure 8. Load Table/Graph Usage Chart}

Analysis of the AC filter usage data indicates the distribution of filter types in form of total counts. Table 3 specifies each of the selected and deselected filter options and shows a total of 3,427 times that AC filter options were used in all three HITLs. This filtering tool was an essential part of both situation assessment and planning. Across the planning teams, the LOAD and TO filters were used the most. The FROM, GEO, VIA, WX, and FL filters were used less frequently (between 5 to 100 times). The DRAW, AIRPORT, ID, and DIR filters were hardly used (less than 5 times). The ability to color-code the filter selections was hardly ever used as well. Feedback from the post-simulation tools questionnaire revealed that the participants thought it would be nice to save the user-created filters along with the default filter settings for each run, and they commented that the filters were easily used in conjunction with the load tables/graphs to identify candidates for reroutes. Even though most of the filter options were scarcely utilized, comments indicated that they liked the idea of the many options available and could see using them with more experience and familiarity with the traffic flows.

Table 3. AC Filter Counts

\begin{tabular}{|l|c|}
\hline \multicolumn{1}{|c|}{ AC Filter Type } & $\begin{array}{c}\text { MSPs and } \\
\text { FAM HITL } \\
\text { Totals }\end{array}$ \\
\hline Selected/Deselected: LOAD & $501 / 272$ \\
\hline Selected/Deselected: TO & $1222 / 881$ \\
\hline Selected/Deselected: FROM & $8 / 8$ \\
\hline Selected/Deselected: FL & $36 / 36$ \\
\hline Selected/Deselected: GEO & $61 / 56$ \\
\hline Selected/Deselected: VIA & $5 / 5$ \\
\hline Selected/Deselected: WX & $95 / 70$ \\
\hline Deselected: All & 171 \\
\hline AC Filter Total & $\underline{\mathbf{3 4 2 7}}$ \\
\hline
\end{tabular}

As aircraft are identified as potential reroutes, the FBTM planning tools were then used to build the new trajectories. A total of 6,244 trial plans were initiated over the 3 HITLs. Of those, 2,724 (44\%) were sent or accepted via Data Comm to either sector controllers or to other planning team members for approval, 2,901 (46\%) were developed but cancelled or never actually sent, and $619(10 \%)$ were suggested trial plans that were opened and just looked at as a result of the user receiving a message from another planning position (magenta arrow in FDB). Figure 9 graphically shows the total combined trial plan usage.

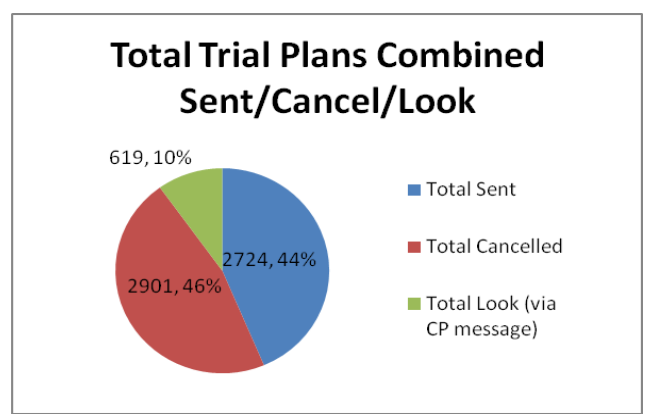

Figure 9. Total Trial Plans Combined 
The planning teams utilized the trial plan tool to not only build new trajectory resolutions but to give themselves more situational awareness. They would open many trial plans just to "assess" impacts before they actually implemented them, thus, the reason for so many cancelled plans.

Figure 10 shows the breakdown of those 6,244 trial plans by their usage types. There are a variety of ways for a planner to build or start a trial plan. (See Table 2 for each method.) The following data will show how the planners actually used the trial plan options. Figure 10 shows the percent of actual trial plan usage broken down by the various trial plan usage options. Typing TT or clicking on the FDB trial plan portal arrow (50\%, 3,130 times) was the most used way of opening a trial plan across all three HITLs. The multi-aircraft trial plan feature (FF) was the second most used method of opening a trial plan. The FF feature data tell how many times it was used and how many aircraft were involved. For example, FF was used for $34 \%$ of all trial plans, 539 times, encompassing 2,119 aircraft ( $n=539,2,119$ aircraft). The third most used method of opening a trial plan was typing TA (9\%, 577 times), typing TR for specific route trial planning $(7 \%, 406$ times) was used the least.

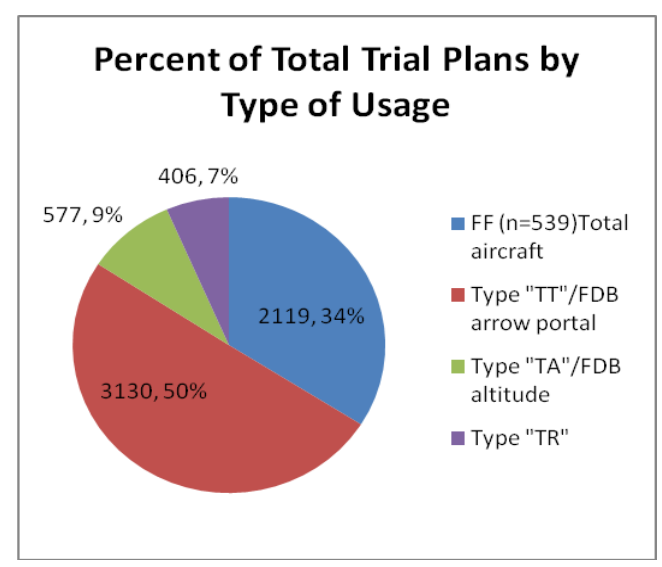

Figure 10. Trial Plan Usage by Type

Comments regarding the FF group trial planning were mostly positive; "great tool to validate all reroutes at once, [I was] able to move multiple aircraft and get immediate feedback on what they were doing to the traffic and the traffic around them, loved the FF function because of the increase in working speed it enabled". On the down side the FF feature was a great function but it was sometimes hard to use. There were too many misses when trying to select an aircraft to be grouped, picking up underlying aircraft when trying to enter a route, and it was to easy to mistype an element and ruin the whole group selection.

Post-simulation tool questionnaire feedback on the general trial plan features (TT or arrow portal) were very positive. The planners used the general trial planning quite often to explore the different routes that could be beneficial to aircraft and sector operations, the feasibility of particular reroutes was established very quickly and it gave a great picture of what those changes would look like and a simple way to tweak the routes to make them work. One user thought it was a great feature but a little too cumbersome to use at times.

Subjective comments on altitude trial planning were that it worked best for closer timeframe solutions as it was very quick and easy to do, and that it was nice to see impact on the customer as well as any conflicts that might occur. The planners liked the practicality of descending aircraft all the way down to low flight levels without having to do all the coordination. However the impact on the lower sectors was not immedialty felt and they wondered about the feasibility of making so many low altitude changes. Other suggestions included when the trial plan altitude was in conflict it was hard to see the other aircrafts altitude, and that the altitude list should show what altitudes are clear and what altitudes would have conflicts.

The route trial planning, TR, was used differently among the planning participants and the comments also reflect those differences. One planner relied heavily on TR to support their plans and solutions, while another used it mostly to see the impact of route changes to other sectors. Also, the third planner mostly used it to move multiple aircraft and get immediate feedback on what they were doing to their traffic and to the trafffic around them. While the fourth planner said that TR is maybe the best tool but the hardest to use. Doing several trial routes and moving the route on some but not all was difficult. Also, when selecting a point on the DSR for the new route, the flight ID of an aircraft underneath or near that point would appear and then have to be deleted.

Coordination planning tools were a very important aspect of all three HITLs as well as to the 
future success of NextGen implementation. Although voice communications, both one-to-one and one-to-many (conference calls) were required for all simulations, only the ground-to-ground and ground-to-air Data Comm usage will be addressed here. $^{2}$ Figure 11 shows the total number of Data Comm types utilized in each HITL.

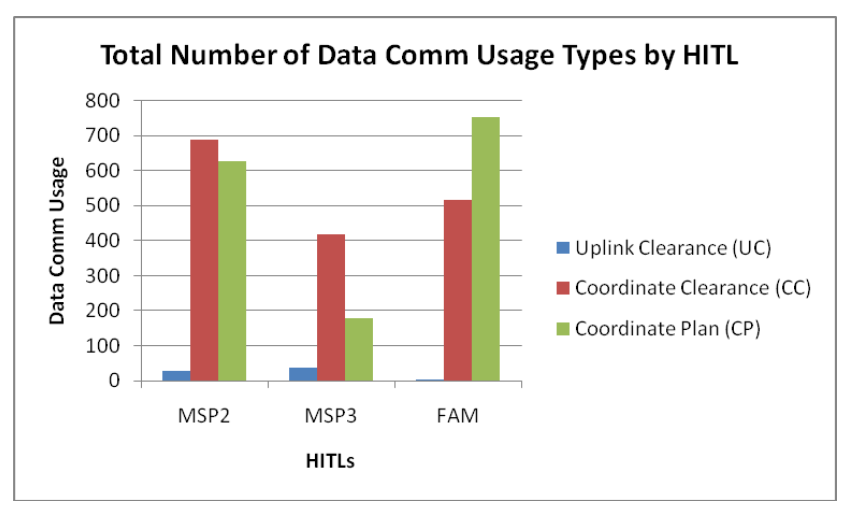

\section{Figure 11. Total Data Comm Usage by HITL}

The UC command uplinks trajectories straight to the aircraft and is usually only used by the radar controllers who have track control of that aircraft. Only 66 UCs were issued across all three HITLs by the Area Supervisors and TMCs as they were only allowed to issue UC clearances when the first trajectory modification point was at least 30 minutes away. The planning team used the $\mathrm{CC}$ message to send their coordinated trial plan clearances directly to the radar controller who has current track control on the aircraft being moved. During the simulations the planning teams sent 1,620 coordinated clearance messages for reroutes or altitude changes to the tactical controller positions. The planning team used the $\mathrm{CP}$ message to send a trial plan suggestion or boundary change solution to other members of the team to get the coordinated plan approved. During the simulations the planning team members sent 1,555 planned trajectory changes to other team members.

In conjunction with all the other FBTM tools, Data Comm was considered a required feature by all the participants. All participants liked the ability to UC a clearance directly to an aircraft, given that there

\footnotetext{
${ }^{2}$ References 16 and 18 give full communication overviews of MSP and FAM HITLs.
}

were operational rules that would need to be defined. The CC and CP capability were deemed necessary to enact ATFM actions amongst all team members and then to be able to send those new trajectory changes straight to the radar controller for implementation was a big advantage over today's operational procedures.

\section{Trajectory Assessment and Modification Tools Usefulness and Usability Data}

In order to support the usage data, postsimulation tools questionnaires were given to each of the 14 ATFM TMCs and Area Supervisors to determine if a specific tool was viable in accomplishing the goals of the simulations. Usefulness and usability data were collected for each FBTM tool. The ratings scale for all useful and usable questions was from 1 to 6 with 1 being Not Very Useful/Usable and 6 being Very Useful/Usable. Results indicate very high useful and usable ratings. See Figure 12 for a complete overview of the data across all three HITLs for the situation assessment tools, trajectory planning tool, and communication and coordination tool sets.

The Load table/graphs were relied on heavily to plan new aircraft routes and decrease sector loads. The table and graphs were rated as very useful and usable for both the situation assessment and planning tasks. One TMC said that the load information was the "bread and butter of working these scenarios" and that it was used constantly for cross-checking any traffic reroutes and boundary changes. Another mentioned that the complexity and load information allowed him to make "decisions not just on the peaks, but the available room in the less busy sectors for possible reroutes".

The AC Filters were rated very useful and usable by all participants. In their comments, Area Supervisor and TMC participants indicated that the filter tool made it easier to provide service for equipage. By filtering out the equipped aircraft and displaying only the unequipped aircraft, participants were able to focus their initial attempts at managing sector complexity on traffic initiatives that only affected the unequipped aircraft, leaving the equipped to remain on their original trajectories. Overall, the AC filters were well accepted and necessary for both situational assessments and trajectory planning opportunities. 


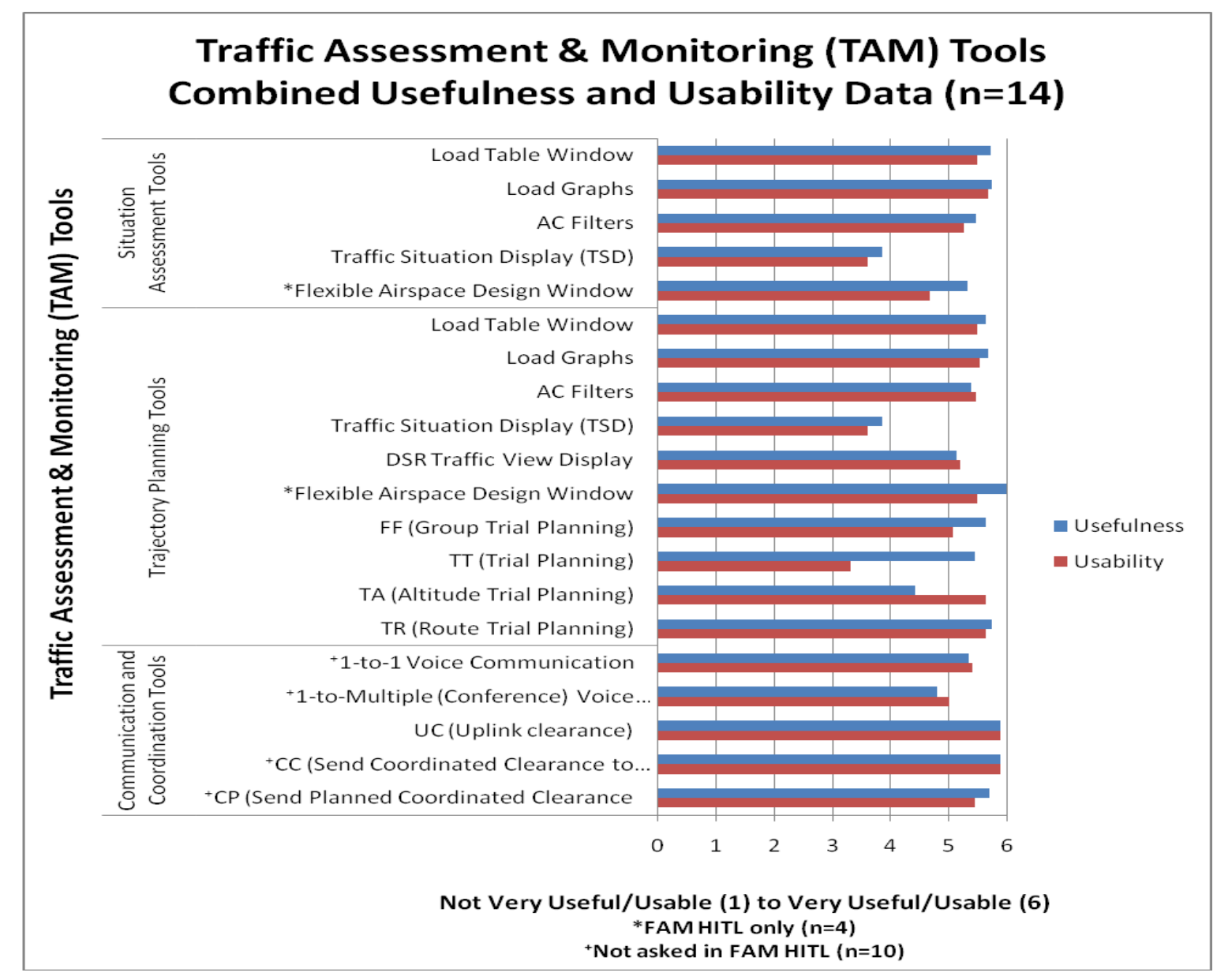

Figure 12. Traffic Assessment and Monitoring Tools Usefulness and Usability Data

The TSD was given a fairly low usefulness and usability ratings by all participants as expected. The full functionality of a fielded TSD was not available and therefore the TSD was provided for information purposes only. The DSR, however, was a highly used tool for trajectory planning and given a high useful and usable rating. Again, the DSR was the primary interface used to view all the traffic and to perform the trial plan re-routing functions. The DSR was essential in conjunction with the load tables, load graphs, and the AC filter to determine if action was needed, and then to identify the appropriate solution. The biggest problem reported with the DSR was the inadvertent picking of underlying or nearby aircraft (target symbols) when trying to click on a route or trial plan portal.

The post-simulation feedback on the trial planning features was mostly positive. The FF (group trial planning) was the second most used trial plan method and TR (specific route trial plans) was the least used. However both were rated highly useful and usable. Even though TT (general trial plan/FDB arrow portal) was rated highly useful and was the most used method of opening a trial plan, it was given a relatively low usability rating. Participants commented that it was sometimes hard to pick the arrow portal on the DSR without getting an underlying FDB making the task more difficult. The TA (altitude trial planning) method was given a higher rating on the usability scale than the usefulness one. Again, feedback suggested that it was easy to use altitude trial planning but that in the case of the long term planning of the TMCs and the controller interaction of the Area Supervisors, altitude is used mostly for controller interaction and more tactical maneuvers, therefore not as useful to the more strategic planning team members. 
Looking at the FAM HITL, and considering that the flexible airspace design window was a first prototype of its kind, the usefulness and usability ratings were quite high. Comments on the boundary editing procedure ranged from "Very easy to understand and highly interactive" and "Good tool, usable", to "[the process] should have been more streamlined, I didn't feel I should have to jump from one box to another and that it felt [like] more of a natural progression". One Supervisor commented that "like most things and ideas, to see them in practice or to work with the tools, provides better comfort and understanding... basically, once we started to use the tools, the better I felt about it, and yes, I like it". Communicating to all the parties involved is a big process for not only FAM boundary change concepts but for all potential aircraft trajectory change solutions.

When trajectory plans or boundary edits were created on one planning position they could be sent to other positions using verbal and non-verbal communication. Figure 12 also details the planner team ratings on usefulness and usability regarding the means of communication. The communication mechanisms were rated mostly positive for both voice and data. Suggestions for improving particularly the exchange and bookkeeping of the (CP) plans between planner positions were made. The communication of trajectories (CCs) to the tactical controller positions was rated mostly useful and usable from the planner positions, while feedback from the sector controllers indicated that some additional explanations and/or annotations would improve the overall communication path.

\section{Discussion}

Collaborative FBTM tools were designed and developed to achieve the overarching goal of integrating core, NextGen components. Situational awareness tools, along with fast and accurate trajectory modification implementation and coordination tools can go a long way towards achieving a system with TBO and CATM capabilities. The usefulness and usability of these tools across all three HITL studies demonstrates how integrated ground systems can support important genres of the NextGen.

By providing common situation assessment and trajectory planning tools to all players in the ATFM team, the FBTM tools provide powerful mechanisms to identify problem areas and allow, informed coordinated decisions to quickly develop. When operators have the same equipment and the same aircraft intent data they have a better, more common understanding of each team member's constraints and a common understanding of the goals. ADS-B technologies in conjunction with air-to-air and air-toground Data Comm will provide the ground systems with improved aircraft intent data needed to continue accurate TBO operations.

Ideally, incorporating FBTM tool-sets into current air traffic operations will lead to a more flexible TBO environment and a more stable CATM solution. These three HITLs and the FBTM tools applied were designed to assess and manage traffic congestion and complexity with most aircraft on user preferred trajectories.

A closer look at the subjective feedback and actual usage data from the operators sheds additional light on interesting aspects of the new tool set. An important goal of these simulations was to evaluate replacing or augmenting a purely traffic count based sector load assessment with complexity parameters. Utilizing the complexity factors instead of straight sector counts allows for more flexibility and increased traffic throughput within ATFM operations.

Flexibility of airspace design and procedures is another important component of NextGen solution sets. The FAM HITL utilizing all the underlying FBTM tools was successful in reducing airspace complexity and increasing aircraft throughput while maintaining a normal level of workload for the controllers. The MSP HITLs followed along the same lines and also demonstrated the feasibility of FBTM tools working in a mixed equipage environment.

\section{Conclusion}

NextGen solutions will have to be integrated with each other. As the FAA's NextGen plan and SESAR move from currently controlled airspace with radar-based air traffic control systems to a more managed airspace with satellite-based systems they will have to quickly leverage and expand on current technologies and implement new automated decision support tools to create a ground based system that can 
work with the advanced aircraft technologies such as ADS-B, performance-based trajectory navigation, air-to-air and air-to-ground Data Comm. FBTM tools such as those developed in the AOL at NASA Ames and tested successfully across different NextGen operational environments can support a more advanced, efficient, safe and flexible air traffic control system.

\section{References}

[1] Federal Aviaition Administration, 2011, FAA Aerospace Forecast Fiscal Years 2011-2031, Washington, D.C.

[2] Federal Aviaiton Administration, March 2011, NextGen Implementation Plan. Washington, DC. www.faa.gov/nextgen.

[3] Federal Aviaiton Administration, 2010, National Airspace and Procedures Plan. Washington, DC. www.faa.gov/nextgen/media/NAPPecopy.pdf

[4] SESAR Joint Undertaking, July 2010, ATM Master Plan Update Working Group Report. Edition 1.1 www.atmmasterplan.eu/http://prismeoas.atmmasterplan.eu/atmmasterplan/faces/public /ur/change_history.jspx

[5] Prevot, T., P. Lee, T. Callantine, J. Mercer, J. Homola, N. Smith, et al., 2010, "Human-in-theloop evaluation of NextGen concepts in the Airspace Operations Laboratory," AIAA-20107609 AIAA Modeling and Simulation Technologies Conference, Toronto, Ontario.

[6] Prevot, T., 2009, NextGen Technologies for Mid-Term and Far-Term Air Traffic Control Operations. 28th Digital Avionics Systems Conference, Orlando FL.

[7] Prevot, T., C. Brasil, M. Mainini, 2010, MultiSector Planning Tools for Trajectory-Based Operations, 10th AIAA Aviation Technology, Integration, and Operations (ATIO) Conference, September 13-15, Fort Worth TX.

[8] Smith, N., P. Lee, T. Prevot, C. Brasil, J. Homola, A. Kessell, H. Lee., M. Mainini, J. Mercer, 2010, A Human-in-the-Loop Investigation of Multi-Sector Planning Operations for the NextGen Mid-Term, submitted to the 10th AIAA Aviation
Technology, Integration, and Operations (ATIO) Conference, September 13-15, Fort Worth TX.

[9] NASA, 2010, Multi Sector Planner Experiment (II) Air Traffic Control and Management Tools Airspace Operations Laboratory Report to FAA, NASA Ames Research Center, Moffett Field, CA.

[10] NASA, 2011, Mulit Sector Planning Mixed Equippage Experiment (III): Air Traffic Control and Management Tools for Mixed Equipage , Addendum to "Multi Sector Planner Experiment (II) Air Traffic Control and Management Tools". Airspace Operations Laboratory Report to FAA, NASA Ames Research Center, Moffett Field, CA.

[11] Smith, N., T. Prevot, C. Brasil, J. Homola, A. Kessell, H. Lee, P. Lee, M. Mainini, J. Mercer, 2011, A Human-in-the-Loop Evaluation of FlowBased Trajectory Manangement in Mixed Equipage Airspace. Presented at the Ninth USA/Europe Air Traffic Management Research and Development Seminar (ATM2011), Berlin, Germany.

[12] Homola, J., P. Lee, C. Brasil, H. Lee, and M. Mainini, 2011, Human-in-the-Loop Investigation of Airspace Design. Presented at AIAA Guidance, Navigation, and Control Conference, Portland, Oregon.

[13] Homola, J., P. Lee, C. Brasil, H. Lee, M. Mainini, and T. Prevot, 2011, Decision Support Tools for Flexible Airspace Management. Submitted to $11^{\text {th }}$ AIAA Aviation Technology, Integration, and Operations (ATIO) Conference. Virginia Beach, VA.

[14] Lee, P.U., C. Brasil, J. Homola, A. Kessell, H. Lee, M. Mainini, N. Smith, and T. Prevot, 2011, Benefits and Feasibility of the Flexible Airspace Management Concept: A Human-inthe-loop Evaluation of Roles, Procedures, and Tools. Presented at Ninth USA/Europe Air Traffic Management Research and Development Seminar (ATM2011), Berlin, Germany.

[15] Lee, P. U., K. Bender, D. Pagan, and K. Carr, 2011, Identifying Functional Requirements for Flexible Airspace Management Concept using Human-in-the-loop Simulations. Submitted to $30^{\text {th }}$ Digital Avionics Systems Conference, Seattle, WA. 
[16] Mainini, M., P. Lee, C. Brasil, H. Lee, J.

Homola, 2011, Flexible Airspace Management

Operator Roles, Task Distribution, and

Coordination Mechanisms. Presented at Human

Factors and Ergonomics Society $55^{\text {th }}$ Annual

Meeting (2011), Las Vegas, NV.

[17] Department of Transportation, 2010,

Federal Aviation Administration Order JO

7210.3W. Facility Operations and

Administration.

http://www.faa.gov/air_traffic/publications/at pubs/fac/1708.html.

[18] Kessell, A, P. U. Lee, and N. Smith, 2010, Ground-Ground Data Communication-Assisted Verbal Communication for Multi-Sector Air Traffic Management, $29^{\text {th }}$ European Association for Aviation Psychology Conference, Budapest, Hungary.

\section{Acknowledgements}

The authors would like to thank the NASA Airspace Systems Programs office and the FAA's NextGen Airspace Project for funding the work on the MSP and FAM HITLs. Special thanks go to the MACS development team for making it all happen and the AOL simulation team for all their efforts.

$30^{\text {th }}$ Digital Avionics Systems Conference

October 16,-20, 2011 